\title{
Epicardial Atrial Activation Assessment from the Surface ECG in Atrial Fibrillation
}

\author{
JJ Rieta ${ }^{1}$, F Hornero ${ }^{2}$, C Sánchez ${ }^{3}$, C Vayá ${ }^{1}$, D Moratal $^{1}$ \\ ${ }^{1}$ Bioengineering, Electronics and Telemedicine. Valencia University of Technology, Spain \\ ${ }^{2}$ Cardiac Surgery Service. General University Hospital of Valencia Foundation, Spain \\ ${ }^{3}$ Innovation in Bioengineering. Castilla-La Mancha University, Spain
}

\begin{abstract}
Atrial fibrillation $(A F)$ is one of the most common complication of cardiothoracic surgery affecting from $30 \%$ up to $60 \%$ of the patients. In this study 15 patients undergoing cardiac surgery, that developed postoperative atrial fibrillation, were selected to assess if the information available in epicardial recordings can be recovered with the only use of body surface recordings. Surface ECGs were processed by independent component analysis (ICA) to extract a unified surface atrial activity $(A A)$ that takes into account the atrial contribution of each lead. Next, the estimated $A A$ has been compared with epicardial recordings, the spectral cross-correlation being $85.34 \pm 11.08 \%$ (range $60.57-$ 97.31) and the average spectral coherence $70.10 \pm 9.46 \%$ (range 54.92-83.95). Therefore, this study has assessed that information provided by the surface ICA-estimated $A A$ is a valid and useful tool to analyze the properties of atrial activation patterns in AF patients, thus allowing to improve the information about atrial arrhythmias in those patients where epicardial recordings are unavailable.
\end{abstract}

\section{Introduction}

Atrial fibrillation (AF) is the most commonly diagnosed sustained arrhythmia in clinical practice [1], affecting up to $1 \%$ of the general population. Considering its prevalence with age, this arrhythmia affects up to $15 \%$ of the population older than 70 [2]. Moreover, there exist evidence that $\mathrm{AF}$ is one of the main causes of embolic events that, in $75 \%$ of the cases, develop complications associated with cerebrovascular accidents, provoking that a patient with $\mathrm{AF}$ can have twice the risk of death than a healthy person with the same age [2]. On the other hand, AF is one of the most common complication of cardiothoracic surgery (coronary bypass or heart valve repair/replacement) [3], affecting up to $60 \%$ of the patients undergoing cardiac surgery, especially in the first days after the intervention, thus increasing morbidity and associated costs [3].
To study AF from surface ECG, atrial activity (AA) and ventricular activity (AV) have to be separated. One solution consist of applying blind signal separation (BSS), which is able to use the multi-lead information provided by the ECG to obtain a unified AA [4]. Considering that the relation between surface and epicardial atrial waveforms is of clinical interest for non-invasive assessment of electrical remodeling in $\mathrm{AF}[5,6]$, it has to be demonstrated clinically if the result obtained via the BSS-based AA estimation methodology is able to offer the same (or similar) information than that provided by atrial epicardial recordings. The present work is focused on clinically assess the similarity and equivalence of both informations and, as a consequence, validate the BSS estimation methodology that, obviously, present the great advantage of being a noninvasive procedure, in contrast with epicardial recordings.

\section{Database}

In this work 15 patients undergoing cardiac surgery that developed postoperative AF were selected. For these type of patients it is usual to attach a set of electrodes on the heart's surface (epicardial electrodes) during the surgery intervention. This will allow a very precise and comfortable postoperative monitoring. Two epicardial electrodes were placed on the right atrium free wall in order to follow those patients that developed AF after the surgery.

For each patient, 12 lead ECGs were recorded during several minutes. Next, one segment of 8 seconds in length was selected for each patient trying to obtain the most significant AF characteristics and avoid patient movements and other disturbances. The sampling frequency was $1 \mathrm{kHz}$ and the resolution of the digital recording system was 16 bits, thus providing a sensibility better than $0.4 \mu \mathrm{V}$. The 12 leads were recorded following standard procedures except for monopolar leads V3 and V4, that were connected to the atrial epicardial electrodes. In this way it was possible to record simultaneously body surface and epicardial activity with the only use of a standard 12-lead ECG system, which is the most popular equipment in clinical cardiology. 
The fact of discarding V3 and V4 from surface recordings has been motivated because AA information is mainly present in leads II, aVF and V1 [1] and, in addition, to record 3-dimensional information of the ECG it is preferred not to discard leads V2 and V5.

The selected segments from each ECG recording have been normalized in amplitude, notch filtered $\left(f_{n}=50 \mathrm{~Hz}\right)$ to cancel out powerline interference, high-pass filtered ( $\left.f_{h}=0.7 \mathrm{~Hz}\right)$ to suppress base line wandering and lowpass filtered $\left(f_{l}=70 \mathrm{~Hz}\right)$ to reduce high frequency noise. All the filtering operations have been performed bidirectionally to avoid phase distortion of the signals. Fig. 1 plots the ECG recording of patient \#14 where the 12 leads in AF can be observed. Note the special particularity of leads V3 and V4 corresponding to epicardial recordings of the right atrium.

\section{Methods}

After the aforementioned preprocessing stage, each ECG recording has been processed with the FastICA algorithm, which is a robust and fast way of solving blind signal separation problems [7]. Next, atrial and ventricular sources have been obtained together with other sources that can be associated to muscular noise [4]. The use of ICA to estimate the AA from ECG surface recordings allows to take into account the atrial contribution in every lead to generate a unified signal estimate condensing the AA information [4]. This procedure of extracting the AA globally can be considered as an improvement in those situations where 12-lead ECGs are available [4, 8,9].

Fig. 2 plots a direct comparison, for patient \#14, between atrial epicardial waveforms, $\mathrm{AA}_{\mathrm{E} 1}$ and $\mathrm{AA}_{\mathrm{E} 2}$, and the estimated AA via ICA from surface recordings $\mathrm{AA}_{\mathrm{S}}$. Next, in order to compare more in depth surface and epicardial signals, spectral analysis has been carried out computing the power spectral density (PSD) with the use of Welch-WOSA modified periodogram. The computations have been performed with 4096-point Hamming windowing, $50 \%$ overlapping between adjacent windows and 8192-point FFTs.

During last years it has been usual to perform AF studies making use of the AA power spectral density to analyze the main atrial frequency and its variations with time or with drug administration $[8,10,11]$. The main atrial frequency is identified with the fundamental harmonic of the arial spectrum, which is associated with the fundamental period of the atrial signal waveform.

Through the study of the fibrillatory frequency it has been demonstrated the possibility of extracting relevant information about the success of electric cardioversion for AF treatment [12]. Because of that, the comparison and estimation parameters defined in this study are focused in the frequency domain, and more precisely, within the band

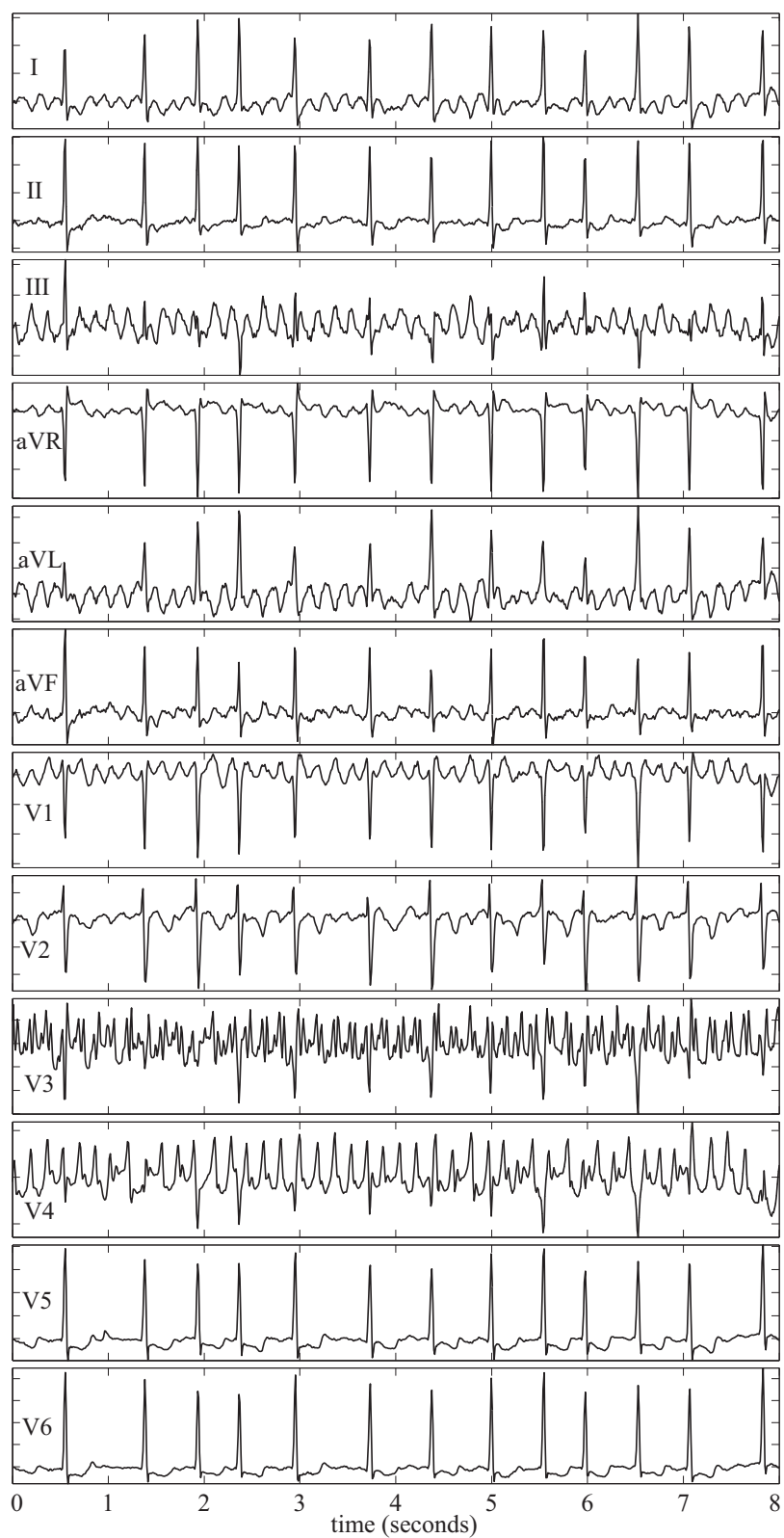

Figure 1. Surface ECG recording of patient \#14. All the electrodes have been placed on their standard position except V3 and V4 which are connected to the right atrium.

of 0 to $20 \mathrm{~Hz}$, which is a wide enough margin to include all the atrial spectral information $[8,10,11]$.

The PSD has been computed both for the surface ICAestimated AA $\left(P_{S S}\right)$ and epicardial recordings $\left(P_{E E}\right)$. In order to establish an additional level of comparison between surface and epicardial activities, the atrial frequency associated to the main atrial peak has been calculated. Fig. 3 shows an example, obtained for patient \#14, of epicardial $\left(P_{E E 1}\right.$ and $\left.P_{E E 2}\right)$ and surface spectra $\left(P_{S S}\right)$. 


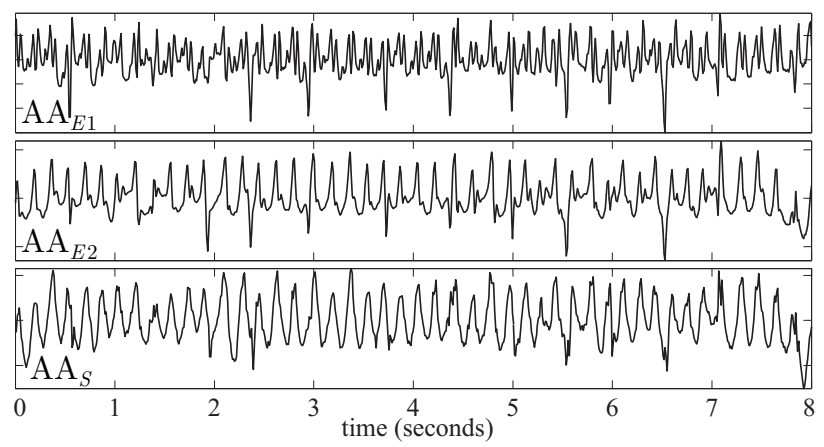

Figure 2. Comparison between recorded and estimated atrial activities. Recordings $\mathrm{AA}_{\mathrm{E} 1}$ and $\mathrm{AA}_{\mathrm{E} 2}$ are from epicardial electrodes and $\mathrm{AA}_{\mathrm{S}}$ is the ICA-based AA estimation from body surface electrodes.

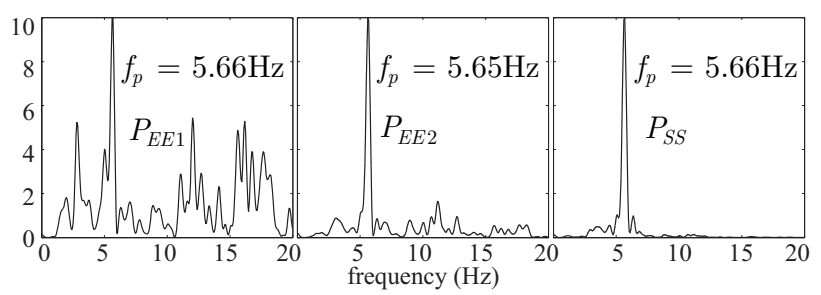

Figure 3. Normalized power spectral density (patient \#14) for atrial epicardial activities, $P_{E E 1}$ and $P_{E E 2}$, and the body surface estimated AA, $P_{S S}$. It is also indicated the main atrial frequency for each case.

Taking as a starting point the PSD, additional spectral parameters have been defined. The spectral crosscorrelation, $\left|R_{P_{S S} P_{E E}}\right|$, is computed as

$$
\left|R_{P_{S S} P_{E E}}\right|=\left|\frac{C_{P_{S S} P_{E E}}}{\sigma_{P_{S S}} \sigma_{P_{S S}}}\right|
$$

where $C_{P_{S S} P_{E E}}$ is the covariance of $P_{S S}$ and $P_{E E}, \sigma_{P_{S S}}$ and $\sigma_{P_{E E}}$ being the standard deviation of the PSDs, respectively. On the other hand, the spectral coherence between epicardial and surface recordings $S_{P_{S S} P_{E E}}$ has been obtained as

$$
S_{P_{S S} P_{E E}}=\frac{\left|P_{S E}\right|^{2}}{P_{S S} P_{E E}}
$$

where $P_{S E}$ is the cross PSD, in other words, the averaged product between surface $\left(P_{S}\right)$ and epicardial $\left(P_{E}\right)$ spectra, respectively. Because the result of spectral coherence is a vector, with the same number of points than the original PSDs involved in its computation, it has been defined an index to evaluate the spectral similarity. This index has been obtained as the average value of the spectral coherence vector in the AA frequency band of interest $(0-20 \mathrm{~Hz})$. For simplicity, this index will also be called spectral coherence from now on.

\section{Results and discussion}

The methodology described in the last section has been applied to the database of patients and the AA has been estimated from surface recordings and compared with epicardial recordings. After the ICA stage, atrial and ventricular components were separated and identified satisfactory in all the cases. For each patient, main atrial frequencies have been obtained both from epicardial and ICAbased surface recordings. In all the cases, a high degree of similarity has been observed between pairs of frequencies, thus corroborating the usefulness and validity of the study. The obtained set of atrial frequencies had normal values, $5.45 \pm 1.27 \mathrm{~Hz}$ (range $3.41-7.69 \mathrm{~Hz}$ ) and the difference between epicardial and surface frequencies is negligible, the average result being $0.026 \pm 0.039 \mathrm{~Hz}$ (range $0-0.125 \mathrm{~Hz}$ ).

With respect to the estimated AA waveform from surface recordings and ICA separation, it has been observed its regularity and the maintenance of the fundamental period associated with the dominant atrial reentry. Of course, this affirmation can be made thanks to the parallel observation of the epicardial recordings. However, some specifical comments have to be made with respect to a concrete epicardial recording.

Firstly, having in mind that an epicardial recording comes from an electrode attached directly to the atrial tissue, it will provide the AA present in that concrete point. Therefore, if the wavefronts of a concrete reentry are unable to reach the point where the electrode is attached to, that AA could be hidden in the recording provided by the epicardial lead or, in other case, that reentry could be masked by other large amplitude reentries present in the same place and time instant. On the other hand, it is also possible the existence of a local reentry very close to the place where the electrode has been attached to. In this case, local reentry will be registered by the electrode but, at the same time, other epicardial electrodes attached to different places could miss or mask this activity because its locality. This fact can also be applicable to the surface ECG.

As a consequence, the signal provided by an epicardial electrode is strongly dependent both on its placement and on the concrete atrial depolarization pattern of that patient and recording instant. Therefore, the epicardial signal variability could be significative. In fact, this can be observed in Fig. 2 where the epicardial plots are monitoring the right atrium of the same patient at the same time. Anyway, the most usual situation will provoke the dominant reentries to be manifested in all epicardial leads. In addition, each lead will present its concrete and local singularities. This fact is the explanation of the differences between epicardial waveforms in Fig. 2 and spectra in Fig. 3. Moreover, in this latter case, the signals $\left(\mathrm{AA}_{\mathrm{E} 1}\right.$ and $\left.\mathrm{AA}_{\mathrm{E} 2}\right)$ present some ventricular contamination and, hence, it is normal to observe additional frequency components than that present 


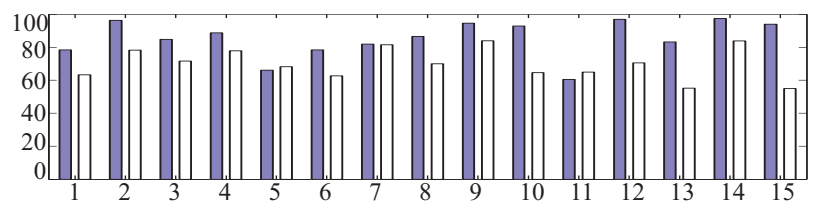

Figure 4. Percentage of spectral cross-correlation coefficient (dark bar) and the average spectral coherence (light bar) for the 15 patients under study.

in the surface ICA-based AA estimation $\left(\mathrm{AA}_{\mathrm{S}}\right)$. Additionally, in all the spectra of Fig. 2 it is possible to observe the existence of a main reentry, with an atrial frequency of $5.66 \mathrm{~Hz}$, clearly showing the maintenance of the fundamental regularity associated to that reentry.

To condense comfortably all the information computed for the patients' database, Fig. 4 plots a normalized bar diagram illustrating the spectral cross-correlation results $\left(R_{P_{S S} P_{E E}}\right)$ and mean spectral coherence $\left(\bar{S}_{P_{S S} P_{E E}}\right)$ between epicardial and surface recordings. The average value of the spectral cross-correlation has been $85.34 \pm 11.08 \%$ (range 60.57-97.31\%) and for the spectral coherence $70.10 \pm 9.46 \%$ (range 54.92-83.95\%). As can be observed in both indexes, results indicate a great similarity between epicardial and ICA-surface information.

\section{Conclusions}

The present study has assessed that atrial activity estimation, using body surface recordings and ICA techniques, is a valid and useful tool to obtain information very close to that provided by epicardial recordings, thus allowing to study atrial activation patterns in AF patients. In addition, the great advantage of ICA to estimate AA is the consideration of multi-lead information and its unification to provide a single AA waveform. This information can be cardiologically relevant in pharmacologic and surgical treatment of AF. Considering the satisfactory results obtained, these techniques could become a useful and valuable tool for atrial activation pattern analysis and reentry study in patients with AF where epicardial recordings are unavailable or where their obtention needs an expensive and uncomfortable procedure.

\section{Acknowledgements}

The authors would like to thank the Cardiac Surgery Service, from the General University Hospital of Valencia (Spain), for the clinical advice provided and kind help in obtaining the signals. This work was partly funded by the research incentive program of the Valencia University of Technology and the action IIARC0/2004/249.

\section{References}

[1] Blomstrom-Lundqvist C, Scheinman MM, Aliot EM, Alpert JS, et al. ACC/AHA/ESC guidelines for the management of patients with supraventricular arrhythmias. European Heart Journal 2003;24(20):1857-1897.

[2] Brembilla-Perrot B, Burger G, Beurrier D, Houriez P, Nippert M, Miljoen H, Andronache M, Khaldi E, Popovic B, de la Chaise AT, Louis P. Influence of age on atrial fibrillation inducibility. PACE Pacing and Clinical Electrophysiology 2004;27(3):287-292.

[3] Crystal E, Connolly SJ, Sleik K, Ginger TJ, Yusuf S. Interventions on prevention of postoperative atrial fibrillation in patients undergoing heart surgery: A meta-analysis. Circulation 2002;106(1):75-80.

[4] Rieta JJ, Castells F, Sanchez C, Zarzoso V, Millet J. Atrial activity extraction for atrial fibrillation analysis using blind source separation. IEEE Transactions on Biomedical Engineering 2004;51(7):1176-1186.

[5] Slocum JE, Ropella KM. Correspondence between the frequency domain characteristics of simultaneous surface and intra-atrial recordings of atrial fibrillation. IEEE Computers in Cardiology 1994;21:781-784, Bethesda, MD.

[6] Husser D, Stridh M, Sornmo L, Olsson SB, Bollmann A. Frequency analysis of atrial fibrillation from the surface electrocardiogram. Indian Pacing and Electrophysiology Journal 2004;4(3):122-136.

[7] Hyvarinen A, Karhunen J, Oja E. Independent Component Analysis. John Wiley \& Sons, Inc., 2001.

[8] Raine D, Langley P, Murray A, Dunuwille A, Bourke JP. Surface atrial frequency analysis in patients with atrial fibrillation: A tool for evaluating the effects of intervention. Jrnl Card Electroph 2004;15(9):1021-1026.

[9] Raine D, Langley P, Murray A, Furniss SS, Bourke JP. Surface atrial frequency analysis in patients with atrial fibrillation: Assessing the effects of linear left atrial ablation. Jrnl Cardiovascular Electrophysiology 2005;16(8):838-844.

[10] Stridh M, Sörnmo L, Meurling CJ, Olsson SB. Characterization of atrial fibrillation using the surface ECG: timedependent spectral properties. IEEE Transactions on Biomedical Engineering 2001;48(1):19-27.

[11] Bollmann A, Kanuru NK, McTeague KK, Walter PF, DeLurgio DB, Langberg JJ. Frequency analysis of human atrial fibrillation using the surface electrocardiogram and its response to ibutilide. American Journal of Cardiology 1998;81(12):1439-1445.

[12] Tai C, Chen S, Liu AS, Yu W, Ding Y, Chang M, Kao T. Spectral analysis of chronic atrial fibrillation and its relation to minimal defibrillation energy. Pace Pacing and Clinical Electrophysiology 2002;25(12):1747-1751.

Address for correspondence:

José Joaquín Rieta

Valencia University of Technology

EPSG Carretera Nazaret-Oliva s/n

46730, GANDIA (Valencia), Spain

E-mail: jjrieta@eln.upv.es 